Canadian

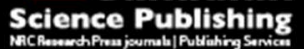

Canadian Journal of Civil Engineering Revue canadienne de génie civil

\title{
A New Collection of Compressed Damage Indices for Multi- Damage Detection of Cold Formed Steel Shear Walls Based on Neural Network Ensembles
}

\begin{tabular}{|r|l|}
\hline Journal: & Canadian Journal of Civil Engineering \\
\hline Manuscript ID & cjce-2015-0417.R1 \\
\hline Manuscript Type: & Article \\
\hline Complete List of Authors: & $\begin{array}{l}\text { zahedi tajrishi, fatemeh; babol university of technology, civil engineering } \\
\text { Mirza Goltabar Roshan, Alireza; Babol University of Technology, Civil } \\
\text { Engineering } \\
\text { zeynalian, Mehran; University of Isfahan } \\
\text { Vaseghi, Javad; Babol University of Technology, Civil Engineering }\end{array}$ \\
\hline Keyword: & $\begin{array}{l}\text { Damage detection, neural network ensemble, frequency response function, } \\
\text { principal component analysis, cold formed steel }\end{array}$ \\
\hline \multicolumn{2}{|c}{} \\
\hline
\end{tabular}




\section{A New Collection of Compressed Damage Indices for Multi-Damage Detection of Cold Formed Steel Shear Walls Based on Neural Network Ensembles}

Fatemeh, Zahedi Tajrishi ${ }^{\mathrm{a}}$, Alireza, Mirza Goltabar Roshan ${ }^{\mathrm{b}}$, Mehran Zeynalian $^{\mathrm{c}}, \mathrm{Javad}$ Vaseghi Amiri ${ }^{\mathrm{d}}$

${ }^{a}$ Ph.D. student, School of Civil Engineering, Babol University of Technology, Mazandaran, Iran, f.zaheditajrishi@stu.nit.ac.ir

${ }^{\mathrm{b}}$ Associate professor, School of Civil Engineering, Babol University of Technology, Mazandaran, Iran, ar-goltabar@,nit.ac.ir

${ }^{c}$ Assistant professor, Department of Civil Engineering, The University of Isfahan, Isfahan, Iran, m.zeynalian@eng.ui.ac.ir

${ }^{\mathrm{d}}$ Professor, School of Civil Engineering, Babol University of Technology, Mazandaran, Iran, vaseghi@nit.ac.ir

\footnotetext{
${ }^{1}$ Corresponding author: Fatemeh Zahedi Tajrishi, School of Civil Engineering, Babol University of Technology, Mazandaran, Iran, Tel:+989122155689, Email: f.zaheditajrishi@stu.nit.ac.ir.
} 


\begin{abstract}
:
This study presents a methodology that utilizes a new combination of two compressed damage indices as input data of an artificial neural network (ANN) ensemble to detect multi-damages in the braces of cold formed steel (CFS) shear walls. To identify an efficient input data for ANN, first, three main groups of damage indices are considered: modal parameter-based damage indices; frequency response functions (FRFs)-based damage indices and time series -based damage indices. Furthermore, principal component analysis (PCA) technique is applied to reduce the dimensions of FRFs and time series -based input pattern. By a sensitivity study, two suitable damage indices of PCA-compressed time series data and PCA-compressed FRFs are identified and then combined together to produce a new efficient input data for a hierarchy of ANN ensembles. The numerical results show that the ANN ensemble-based damage detection approach with the proposed collection of two damage indices is effective and reliable.
\end{abstract}

Keywords: Damage detection; neural network ensemble; frequency response function; principal component analysis; cold formed steel; strap brace; time series; damage index; shear wall. 


\section{Introduction}

Structural damage detection has become an important research topic for evaluation of structural systems safety during their lifetime. In recent three decades various methods have been developed to increase the efficiency and reliability of damage detection approaches. The process of damage detection consists of three main stages as detecting the (1) existence, (2) location and (3) extent of damage in a structural system (Rytter 1993). As damage detection is an inverse, nonlinear and non-unique problem, the conventional mathematically- based engineering techniques (i.e., hard-computing methods) are not very efficient in solving such problems (Ghaboussi and $\mathrm{Wu}$ 1998). In recent years, there has been an extensive effort to determine accurate damage detection techniques. Different soft computing methods including a variety of neural networks, linguistic- based methods (such as fuzzy logic), evolutionary computational models (such as genetic algorithms), and some hybrid algorithms proved to be more effective in solving damage detection problems, especially in vibration-based damage detection techniques (Koh et al. 2003). Artificial neural network (ANN) is a powerful tool for solving damage detection problems because of the pattern-matching, self-adaptiveness, generalisation, and abstraction capabilities(Hansen and Salamon 1990). ANNs and ANNs ensemble have been widely reported in research to estimate damage location and severity in structures (Bakhary et al. 2007; Bakhary et al. 2010; Dackermann et al. 2010).

In order to investigate the efficiency of the ANN- based damage detection approaches, various structural properties have been examined as input to ANN for damage detection of different structural systems. Szewczyk and Hajela (1994) used static parameters under predefined loads to demonstrate the performance of an ANN in damage detection of truss and frame structures. Barai and Pandey (1995) introduced static parameters as input to an ANN for damage detection in 
bridge truss configuration. Sahin and Shenoi (2003) used a combination of global (changes in natural frequencies) and local (curvature mode shapes) vibration-based analysis data as input in an ANN for damage detection of beam-like structures. Lee et al. (2005) presented a damage detection procedure in which the differences or the ratios of the mode shapes between before and after damage had been used as the input to the ANN. Mehrjoo et al. (2008) presented a method to estimate the damage intensities of joints for truss bridges using a neural network with incomplete measurements of the mode shapes as inputs. Fang et al. (2005) used Frequency Response Functions (FRFs) as input data to a neural network in order to detect the severity and location of cracks in beam-like structures. Jeyasehar and Sumangala (2006) studied the feasibility of using an ANN trained with only natural frequency data to detect the damage in pre-stressed concrete beams. Zang and Imergun (2001), Ni, Zhou et al. (2006) and Li et al. (2011) applied a principal component analysis (PCA) - based data reduction technique to the measured FRFs as the input variables of ANN instead of the raw FRF data. Shu et al. (2013) studied the feasibility of using the changes of variances and covariance of the dynamic responses of the structure as input to an ANN for damage detection of a railway bridge.

This paper presents a new input pattern for multi-damage detection of the braces in strap-braced cold formed steel (CFS) shear walls based on ANN ensemble. For this purpose, first, various vibration-based damage indices are considered as input data to ANN in three main groups: 1modal parameter-based damage index; 2-FRF-based damage index; and 3- time series -based damage index. Furthermore, to obtain the suitable input data for network training, the FRFs and time series data are compressed to a few PCs adopting PCA techniques. In order to check the robustness of the selected damage indices as input data to ANN, artificial random noise has been generated numerically and added to noise-free data during the training of the ANNs. Then the 
efficiency of each damage index as input data for ANN is investigated via a sensitivity analysis. PCA- compressed FRFs and PCA- compressed time series are identified as two suitable damage indices. Finally, to improve the results, neural network ensembles are introduced and a new collection of PCA- compressed FRFs and PCA- compressed time series dataare defined as input data to ANN ensembles. The effectiveness and reliability of the proposed method is tested and validated on the numerical data of a CFS shear wall. The remainder of the paper is organized as follows. Section 2 introduces the theoretical background of three groups of damage indices as well as the PCA technique. Section 3 gives a detailed description of the adopted approach. A sensitivity analysis is conducted in section 4 and the conclusions are summarized in section 5 .

\section{Theoretical Background}

Vibration-based damage detection methods are the global techniques based on the principle that the damage changes both the physical properties, such as mass, stiffness and damping, and the dynamic properties of a structure. Therefore, by extraction of dynamic features from the structural vibration, damage can be identified. In general, there are three types of measured dynamic features known as damage indices: modal parameter-based damage indices, frequency response function (FRF)-based damage indices and time series -based damage indices. A description of each damage index considered in this paper as well as PCA technique for data reduction is presented as follows.

\subsection{Modal Parameter-Based Damage Index}

Traditionally, modal parameters such as natural frequencies, mode shapes and damping ratios are the most common dynamic features used for vibration-based damage detection. The resonant 
frequencies were specially used as the damage feature in the early years of vibration-based damage detection (Adams et al. 1978). Unfortunately, in several damage detection cases, the resonant frequencies have been found to be insensitive to the structural damage, especially to the lower levels of damage (Chen et al. 1995; Farrar et al. 2001; Trendafilova 2005). Chen et al showed that the information of the natural frequency change is insufficient to be a useful indicator of structural safety (Chen, Spyrakos et al. 1995). Furthermore, low sensitivity of frequency shifts to damage requires either very precise measurements or large levels of damage (Farrar et al. 2001).. For field application, another drawback is that the natural frequencies are heavily dependent on environmental condition such as temperature or humidity fluctuations (Kim et al. 2007). The mode shapes obtained before and after damage can be either directly used or indirectly used as damage index. Modal strain energy differences (Dackermann et al. 2010) is one of the examples of modal parameter- based damage index used to identify the structural damage. In comparison with frequency methods, mode shape methods are less sensitive to environmental changes and provide better results for both the damage location and severity identification. In contrast, mode shape- based methods are often very sensitive to the incompleteness of the measured data and therefore require measurements from a large number of sensors to ensure the reliability of results. In addition, to extract mode shapes, a data processing procedure known as experimental modal analysis is required. Experimental modal analysis could be less reliable when the measured data are noise contaminated (Li et al. 2011).

\subsection{FRF-Based Damage Index}

Frequency response function (FRF) is a non-dimensional quantity that expresses how vibration is transmitted between the points of a structural system as a function of frequency. FRF data are 
one of the easiest dynamic features to extract since they are directly measured and they require only a small number of sensors and very little human involvement (Tang 2005). Compared with modal parameters, FRF requires much less data processing which reduces the adverse effects of environmental condition and operational condition in damage detection process. Utilizing a combination of FRFs and ANNs for the structural damage detection has shown promising results (Zang and Imregun 2001). However, there is a major drawback in application of ANN using FRF data as input due to the large size of the FRFs. Utilizing full size FRFs as input data to ANN will result in a large number of input nodes, which will cause problems in training convergence and computational efficiency. If only partial sets of FRFs are considered, an improper selection of data points from frequency windows will cause the loss of important information and therefore the damage detection results may be unreliable (Ni et al. 2006).

\subsection{Time Series -Based Damage Index}

The use of time series analysis techniques for damage detection has been extensively explored by several researchers ( $\mathrm{Li}$ and Yang 2008). One of the statistical properties which can be used as input data for ANN is the covariance of the vibration response as Eqn 1

$\operatorname{Cov}\left\lfloor\left(x_{i}(t), x_{j}(t)\right\rfloor=f_{m}(K, C, F, \Phi)\right.$

where $\operatorname{Cov}()$ is the covariance of time series data, $x_{i}(t), x_{j}(t)$ are displacement or acceleration

responses of the system at measurement points of $i$ and $j . f_{m}$ is the covariance relationship function, $\mathbf{K}$ is the matrix of structural stiffness, $\mathbf{C}$ is the matrix of coefficient of viscosity, $\mathbf{F}$ is the external force and $\Phi$ is the matrix of modal shape. Eqn 1 shows that the change of the covariance is sensitive to the change of the structural stiffness of the system (Shu et al. 2013). By sensitivity analyses on the single-degree of freedom system and multi-degree of freedom system, 
$\mathrm{Li}$ and Yang investigated that the variances (covariance) of displacement and acceleration are related to the structural stiffness while the variance of velocity has no robust relationship to the structural stiffness. (Li and Yang 2008). Therefore, the changes of covariance of vibration responses before and after damage can be used as time series data-based damage index.

\subsection{PCA Technique}

To obtain suitable input data for ANN, the input data with the large size (i.e. FRFs or time series data) can be compressed to a few variables with a statistical data reduction technique and then the compressed input data are fed to the neural network. Principal component analysis (PCA) is a statistical technique which reduces the size of data and therefore can be used for feature extraction. By projecting data onto the most important principal components (PCs), its size can greatly be reduced without significantly affecting the data. In structural dynamics, PCA has been applied to measure vibration signals for reduced-order modeling, parameter identification of nonlinear systems, modal analysis and elimination of the influence of environmental effects. The application of PCA for vibration-based damage detection was first proposed by Worden et al. (Worden, Manson et al. 2000) and has been since studied by a number of papers (De Boe and Golinval 2003; Kullaa 2003; Ni et al. 2006).

For a data set $\left[\mathrm{X}_{\mathrm{ij}}\right]$ with $(i=1,2, \ldots, m)$ and $(j=1,2, \ldots, n)$, the mean $\bar{x}_{j}$ and the standard deviation $s_{j}$ of the $j$ th column are obtained as

$$
\begin{array}{r}
\bar{x}_{j}=\frac{1}{m} \sum_{i=1}^{m} x_{i j} \\
s_{j}=\sqrt{\frac{\sum_{i=1}^{m}\left(x_{i j}-\bar{x}_{j}\right)^{2}}{m}}
\end{array}
$$


where $m$ is the total number of observations and $k$ is the dimension (variables) of the observations. Then, the data set $[\mathrm{X}]$ is transformed into the standard normal space yielding the variation matrix $[\tilde{X}]$. A normalized element $\tilde{x}_{i j}$ is expressed by Eqn 4

$$
\tilde{x}_{i j}=\frac{x_{i j}-\bar{x}_{j}}{s_{j}}
$$

The covariance matrix $[\mathrm{C}]$ is given as Eqn 5.

$$
[C]=\frac{[\tilde{X}]^{T}[\tilde{X}]}{m-1}
$$

Finally, by eigenvalue decomposition of the covariance matrix [C], the PCs are obtained as Eqn 6

$[C]\left\{P_{i}\right\}=\lambda_{i}\left\{P_{i}\right\}$

where $\lambda_{i}$ is the $i$ th eigenvalue and $\left\{P_{i}\right\}$ is the corresponding eigenvector. The first PC, which is the largest eigenvalue and its associated eigenvector, denotes the direction and the amount of the maximum variability of the original data set. The second PC, which is orthogonal to the first PC, denotes the second most significant contribution from the original data set, and so on. The most significant PCs represent the most dominant features from the data set. The dimension of the original data set can greatly be reduced by eliminating components that contribute least to the overall variance (Zang and Imregun 2001).

\section{Adopted Approach}

This paper investigates a robust methodology to identify damage severities in the braces of CFS shear wall using a collection of two compressed damage indices and neural network ensembles. To obtain suitable input data for network training, three main groups of damage indices are considered and PCA technique is applied. To simulate field-testing conditions, white Gaussian noise is added to the numerical data and a limited number of sensors are considered. Then, two 
suitable damage indices are chosen among the various damage features via a sensitivity analysis. To improve the efficiency and reliability of the damage detection process, a collection of the two suitable damage indices are introduced as input data for ANN. Furthermore, a hierarchy of neural network ensembles is adopted to respect the different characteristics obtained by individual measurements from the suitable damage indices and different sensor places. Finally, a neural network ensemble fuses the outputs of the individual networks and an overall damage detection is obtained. The whole procedure is divided into the following parts.

1-Finite element (FE) modeling of the CFS shear wall and damage scenarios introduction.

2- Extraction of the dynamic features of the wall in three groups of modal parameter-based damage features, FRF-based damage features and time series -based damage features.

4- Adopting PCA technique to reduce the dimensions of FRFs and time series data.

3- Developing neural network for each damage index.

4- Sensitivity study to investigate the proper damage indices.

5- Introducing a collection of two suitable damage indices and neural network ensembles.

6-Testing and validating the proposed method.

Each part is discussed in details as follows.

\subsection{Finite Element Modeling}

The shear wall considered for damage detection in this study is a 2.4 to $2.4 \mathrm{~m}$ strap-braced CFS wall (Zeynalian and Ronagh 2012). Figure 1 shows the general configuration of the shear wall. All the frame elements, such as top and bottom tracks, studs and noggins were made from an identical C-section of dimensions $90 \times 36 \times 0.55 \mathrm{~mm}$. All studs were connected together at each 
flange by one rivet. In order to increase buckling capacity of the chords and studs, a noggin was used to shorten the buckling length of the vertical members. Solid X straps were connected to the corners of the frame on both sides. The dimensions, cross section and material properties of the C corners section and straps are presented in Tables 1 and 2, respectively (Zeynalian and Ronagh 2012).

The finite element (FE) model representing the frame with the geometry and structural properties corresponding to the specimen described is created using commercial software, OpenSees. As shown in Figure 2, the studs, tracks and noggin are modeled by elasticBeamColumn element and strap braces are modeled by truss element with ElasticPPGap material which can model the tension only braces (Mazzoni et al. 2006). In order to reduce FE model error for robust damage detection, it is necessary to validate the accuracy of the presented numerical model with the experimental test. Figure 3 shows the reasonable agreement between experimental and numerical elastic pushover curves obtained from experimental test and elastic pushover analysis, respectively.

Damage cases are introduced by reduction in structural stiffness of the braces. Structural stiffness reduction is modeled by a reduction in modulus of elasticity $(\mathbf{E})$ of each strap. The incremental reduction in $\mathbf{E}$ is chosen as $0 \%$ (healthy) up to $80 \%$ local damage. Therefore local damage is applied with 9 damage severities at 2 different braces i.e. 81 different damage scenarios as listed in Table 3.

Each damage scenario is indicated by a vector of SSR. SSR vector contains two parameters representing the structural stiffness ratio of each brace as shown in Eqn 7

$$
S S R=\left[\frac{E_{D 1}}{E_{H 1}}, \frac{E_{D 2}}{E_{H 2}}\right]
$$


where $E_{D i}$ and $E_{H i}$ are modulus of elasticity of the $i$ th brace in the damage case and the healthy case, respectively.

\subsection{Input Data to ANN}

The damage features used as input data to ANN are considered as 1- modal parameter-based input data, 2- frequency response function (FRF)-based input data, 3- time series -based input data, and 4- PCA-compressed input data.

\subsubsection{Modal Parameter-Based Input Data}

In order to provide natural frequencies and mode shapes of the shear wall as modal features, the modal analysis is carried out using Eigen analysis in OpenSees (Mazzoni et al. 2006). The first two natural frequencies of the healthy frame corresponding to the lateral mode shapes of the general shear wall are given in Table 4 . The table also shows the reduction in natural frequencies corresponding to four different damage severities of two braces. The levels of damage severity are considered as low $(\mathrm{SSR}=[0.85,0.85])$, medium $(\mathrm{SSR}=[0.45,0.45])$, high $(\mathrm{SSR}=[0.25$, $0.25])$ and variable $(\mathrm{SSR}=[0.35,0.75])$. The horizontal components of the first two lateral mode shapes are measured at two measuring points as shown in the Figure 2. The number of measuring places is kept as few as possible and the measuring points are chosen based on the observation ability of mode shape curves. The mode shape ratios (MSR) before and after damage at two measurement locations for aforementioned damage severities are denoted by MSR in Table 4. It can be seen that the two natural frequencies and mode shape components are affected to different extents due to the structural stiffness ratio (SSR) of the braces. Therefore they are used to make the first two damage indices for ANN-based damage detection of the CFS frame; 1- natural 
frequencies of the first two lateral mode shapes (NF), 2- natural frequencies and horizontal component ratios of the first two lateral mode shapes (NF+MSR). The first damage index is considered to investigate the ability of only natural frequency for damage detection. The second one is considered based on the research showing that the ratio of the mode shapes are more capable than the mode shapes themselves (Lee, Lee et al. 2005). White Gaussian Noise with zero mean and specified standard deviation is introduced to mode shape data to simulate measurement errors and ambient noise. Three different noise pollution levels (1, 3 and 5\% noise-to-intact numerical data ratio) are applied in this study. For each level of noise, three sets of noisecontaminated modal data are generated as input data of ANN.

\subsubsection{FRF-Based Input Data}

In order to provide features in the form of FRF data, transient analysis is performed under impulse loading and time history responses are generated by OpenSees. An impact horizontal force of $800 \mathrm{~N}$ is applied at the impact point (depicted in Figure 2) and the horizontal accelerations of the frame are recorded at two measuring points as response time history data. To further simulate field conditions, white Gaussian noise of three intensities (1, 3 and 5\% noise-tosignal ratio) is added to both, the input impact force signals and the response time history data. For each level of noise, three sets of noise- contaminated data are generated.

In the next step, the noise- contaminated time history data are transformed into the frequency domain using the fast Fourier transform (FFT). By FFT, first, the impact force signals $f(\omega)$ corresponding to frequency $\omega$ and the response signals from horizontal accelerometers $g(\omega)$ are obtained as inputs and outputs, respectively. Next, the FRFs denoted by $H(\omega)$ are estimated by Eqn 8 
$H(\omega)=\frac{G_{g g}(\omega)}{G_{f g}(\omega)}$

where $G_{f g}(\omega)=f(\omega) \times g(\omega)$ is the cross-spectrum between the input and output and $G_{g g}(\omega)=g(\omega) \times g(\omega)$ is the auto-spectrum of the output.

In order to extract FRF-based damage features, a frequency range of $0-512 \mathrm{~Hz}$ is captured. Each FRF comprises of 512 spectral lines with a frequency resolution of $1 \mathrm{~Hz}$ per data point. To determine pattern changes caused by damage, residual FRFs, which are the FRF differences between the healthy and the damaged frame, are calculated. For each noise level a total of 243 residual FRFs are generated by comparing each noise-polluted undamaged case with each of the noise-polluted damaged cases ( 81 damage scenario $\times 3$ noise-polluted data sets).

Figure 4 shows FRF summation graphs obtained by adding up the FRFs of two measuring points in the frame for the healthy and various damage states. It can be seen that by increase in damage severity, FRF peaks change in amplitude and position. In general, a complex pattern of FRF changes such as shifting peaks and altering amplitudes which relate to different damage scenarios is observed. Therefore, residual FRFs, which are defined as differences between FRFs of damaged and healthy frame (Eqn 9) can be utilized as FRF-based damage index

$H_{\operatorname{Re} s}(\omega)=H_{d}(\omega)-H_{h}(\omega)$

where $H_{\mathrm{Res}}(\omega)$ is residual FRF, $H_{d}(\omega)$ and $H_{h}(\omega)$ are FRFs of damaged and healthy frame respectively.

Although FRFs are sensitive to structural damage, they have many obstacles such as the large size and complexity of data. In practice, usage of all available FRF data as an input to ANN may cause problems in the training and convergence process. Therefore, to condense the FRF-based damage feature, a damage index termed as the compressed normalized residual FRF (CNR-FRF) 
is established(Dackermann et al. 2010). Here, only the residual FRF values around the frequency peaks with a frequency bandwidth of $10 \mathrm{~Hz}$ are considered. In addition, the residual FRFs of each selected frequency range are normalized by their maximum value. Thus, the CNR-FRF is considered as the FRF-based input data to ANN in this study.

\subsubsection{Time Series -Based Input Data}

In order to provide a suitable input data for ANN from time series responses, firstly, transient analysis is performed with OpenSees under impulse loading of $800 \mathrm{~N}$ and then the time history responses of the frame are obtained at two measuring points in terms of accelerations. White Gaussian noise of three intensities (1, 3 and 5\% noise-to-signal ratio) is added to the response time history data and three sets of noise- contaminated data are generated for each level of noise. The changes of covariance of noise- contaminated time history accelerations before and after damage are then investigated. Figure 5 shows the relationship between the changes in the covariance and damage severity for each of 81 damage severities. For conciseness, only the changes of the variance at the measuring point 2 are shown here. It is observed from the figure that the damage index changes as expected with the increasing damage severity. Therefore, the changes of covariance of noise- contaminated time history accelerations before and after damage are considered as the time series -based input data denoted by COV-TSD(Shu et al. 2013).

\subsubsection{PCA-Compressed Input Data}

To produce the damage indices that are suitable for neural network training, the dimension of the damage features with the large size must be reduced. For example, a full-size residual FRF of one measuring point, which covers a frequency range of $0-512 \mathrm{~Hz}$, contains 512 input nodes in 
the neural network. Such large numbers of input points cause severe problems in training convergence and computational inefficiency. Therefore, in such applications, PCA is desirable to reduce the size in addition to filter noise (Dackermann et al. 2010).

To compress the input data in this study, two matrices are formed, i.e. one for residual FRFs and one for time series data (TSD) and are subsequently projected onto its PCs utilizing the 'princomp' function in MATLAB. The rows of the matrices comprise of the samples of the damage severities. These are the 243 of the CFS frame data, which contain the 81 damage cases with three sets for each of 1,3 and 5\% noise intensities. The columns of the matrices are the 512 or 1000 input nodes of the residual FRFs or the acceleration responses, respectively. After projection, each sample is represented by 512 or 1000 PCs, respectively. A Plot of the individual and the cumulative contributions of the first 50 PCs of the residual summation FRFs of the CFS wall are shown in Figure 6. It is found that the first 50 PCs account for $99.98 \%$ contribution of the original data for the FRF. Thus, it seems reasonable to choose the first 50 PCs as the most significant input data of the neural networks.

\subsection{Application of ANN}

To design an effective neural network for damage detection of strap braces, six damage indices are extracted as follows:

1- Natural frequencies (NF)

2- Natural frequencies and mode shape ratio (NF+MSR)

3- Compressed normalized residual-frequency response function (CNR-FRF)

4- Covariance of time series data (COV-TSD)

5- PCA-compressed FRF (PCA-FRF) 


\section{6- PCA-compressed TSD (PCA-TSD)}

These damage features are utilized as input data to the neural networks to estimate the damage severities of strap braces. For each damage index, first, individual neural networks are created. Each individual network is supervised feed-forward back-propagation network which consists of an input layer, one hidden layer, and an output layer as shown in Figure 7. The numbers of neurons of input layer represent the numbers of nodes of damage index as input data. The numbers of neurons for hidden layer are determined via trial training. The output layer of each network consists of one single node estimating the structural stiffness ratio of each brace denoted by SSR in Eqn 7. Hyperbolic tangent sigmoid functions are utilized as nonlinear activation functions for the layers of each ANN. The training is performed utilizing the back-propagation conjugate gradient descent algorithm. In order to maintain the generalization ability of the network, the input data is randomly divided into three sets; a training, a validation and a testing set and then the early-stopping method is applied during the training. The ratio of input data vector for training is set to 0.6 and the ratio of input data vector for each of validation and testing is set to 0.2 . In the early-stopping method, while the network is trained with the training set, its performance is supervised by the validation set to avoid overfitting. The network training stops when the error of the validation set increases while the error of the training set still decreases, which means that overfitting occurs and the generalization ability of the network is lost. Details of information of the designed networks are listed in Table 5. The design and operation of all neural networks is performed with the software MATLAB. 


\section{Results and Discussion}

\subsection{Damage Index Sensitivity Study}

First, neural networks are trained with the defined six damage indices to identify damage severities of straps. Then, in order to establish a measure of capability of each ANN for damage detection, the normalized error of the damage quantification of each strap brace is defined as

$E_{\text {norm }}(d)=\frac{T_{d}-O_{d}}{S_{\max }}$

where $d$ and subscript $d$ indicate the damage scenarios, $T_{d}$ the target value of $d, O_{d}$ the network output value of $d$, and $S_{\max }$ the maximum severity of a damage (here $80 \%$ loss of the modulus of elasticity, E). Therefore, a band ranges from $-6.25 \%$ to $+6.25 \%$ normalized error around the $0 \%$ error is introduced to represent the area in which the network estimations must fall in order to correctly identify the damage $\left(E_{\text {norm }}(d)=\frac{T_{d}-O_{d}}{S_{\max }} \rightarrow E_{\text {norm }}(d)=\frac{ \pm 5}{80}= \pm 0.625\right)$. Then, for each of six damage indices, the percentage of false outputs of each strap brace is evaluated for three levels of noise pollution. The false detection is defined as the ANN output which its $E_{\text {norm }}$ is out of this band $\left(E_{\text {norm }}(d) \geq+6.25 \%\right.$ or $\left.E_{\text {norm }}(d) \leq-6.25 \%\right)$. The numbers of false detections normalized by the numbers of all detections for each individual ANN is defined as the percentage of false output. Furthermore, the maximum and average of absolute normalized error of the damage quantification of each strap brace for each of six damage indices are evaluated for three levels of noise pollution. Summaries of the damage detection results of the networks for each of strap braces are shown in Tables 6-11. In the tables, the percentage of false damage detection along with the maximum and average of absolute normalized error of the damage quantification of two braces are listed for each of six damage indices and three levels of noise pollution. 
From the tables, it is visible that almost all damage cases of three noise pollution levels are falsely estimated by modal parameter-based neural networks. In addition, these ANNs have the highest values of maximum and average of absolute normalized error. Therefore, the modal parameters are not adequate as input for neural network-based damage detection of the CFS wall. The tables also indicate that almost half of damage cases cannot be identified correctly by CNRFRF and COV-TSD. In contrast, PCA-FRFs and PCA-TSD show the lowest percentage of false detections as well as acceptable values of normalized error. Although the estimations from networks trained with these two damage indices give false predictions of some damage cases, they are selected as two more suitable damage indices which can be utilized as input data to neural network ensembles. From the tables it could be concluded that the ANN trained with the proposed damage index of PCA-TSD in this research has more accurate results than the ANN trained with the common damage inex of PCA-FRF.

\subsection{Neural Network Ensembles}

From the results above, it can be observed that it is problematic for damage detection when relying on the outcomes of the individual networks trained with the first four aforementioned input data. In addition, training networks with the PCA- FRFs and PCA-TSD improves but does not give the best results. To achieve more reliable damage detection, a conclusive, intelligent fusion of the individual network outcomes is necessary. This can be achieved by a neural network ensemble, which combines the outcomes of the individual networks (Hansen and Salamon 1990).

For this purpose, first, four individual networks corresponding to PCA-FRF and four individual networks corresponding to PCA-TSD are designed. The first two of four individual ANN trained 
with the data from two measurement points, the third one is summarized data that are obtained by adding up the data from two individual measurements and the fourth one is comprised of the whole input data of three other networks. Then, the outcomes of the eight individual neural networks are fused by a neural network ensemble. Through assembling the eight neural networks, the generalization ability of the neural network system can significantly be improved (Zhou et al. 2002) and an overall damage prediction is obtained. The neural network ensemble model is shown in Figure 7 and a plot of the adopted approach is presented in Figure 8.

The network ensemble is designed with eight input nodes, which are the outputs of the eight individual networks; one hidden layer consisting of 25 nodes and one output node predicting the damage severity of each strap brace.

For the neural network ensemble, the percentages of false detection along with the maximum and average values of absolute normalized error of the damage quantification of two braces are listed in Tables 12 and 13 for three levels of noise pollution. To evaluate the outcomes of the network ensemble more closely; the results of damage identification for three noise levels are displayed in Figure $9(\mathrm{a}-\mathrm{h})$. In these figures, the horizontal axis displays the damage scenarios that are sorted by their numbers as depicted in Table 3 . The vertical axis represents the normalized error of the damage quantification of each strap brace. A marked band around the $0 \%$ error axis represents the area in which the network estimations must fall in order to correctly categorise the damage. For the quantification of damage, the band ranges from -6.25 to $+6.25 \%$ normalized error, representing the mid points in-between two damage severities.

Figure $9(a-b)$ along with the Table 12 show that the neural network ensemble trained with a collection of two compressed vibration data is capable of identifying the severities of all damage cases precisely for noise level of $1 \%$. Figure $9(\mathrm{c}-\mathrm{d})$ and the Table 12 indicate the outcomes of 
accurate damage identification by the network ensemble for all damage cases at $3 \%$ noise pollution level except $3 \%$ of damage cases for brace 2 . As expected, the accuracy of damage detection decreases as the noise level increases. As it is observed in Table 12 and Figure 9(e-f), the outcomes of damage identification for $5 \%$ noise pollution have some errors for a number of damage scenarios. However, the percentage of false detections are less than $8 \%$ and the maximum value of absolute normalized error is less than $15 \%$, which means that the method can identify the damage with reasonable accuracy for the high level of noise. The outcomes of final damage identification clearly show the efficiency of the network ensemble trained with two compressed vibration data. The outcomes of the ensemble provide more accurate results than those of the two former neural networks. These results approve the results of previous researches about the efficiency of ANN ensemble for structural damage detection. Although the capability of ANN ensemble has been proved in previous researches, this paper has a new achievement in the field of ANN ensemble which is based on the combination of two different damage indices. The results show the high ability of combination of two different damage indices which have not been combined together in the previous researches.

\section{Conclusion}

This paper presents a thorough investigation of a vibration-based damage detection technique, which utilizes a collection of compressed vibration data in combination with neural network ensembles, to identify severities of damage in strap-braced cold formed steel shear wall. PCA techniques are adopted to extract damage indices and to reduce the dimension of measured FRF data and time series responses. Only the most significant PCs are used as input data to neural network ensembles. White Gaussian noise of up to $5 \%$ noise-to-signal-ratio is added to 
numerical data to simulate uncertainties and the noise robustness of the developed method is investigated by a noise sensitivity study. The main findings of the paper are listed as follows:

-In comparison with modal parameter-based damage detection approach, the proposed methodology has the capability to cope with incomplete FRFs and time series data extracted from a limited number of sensors. The proposed method not only requires much less postprocessing on the measured data, but also yields more reliable damage predictions.

-The damage detection results of two individual ANNs trained with PCA-TSD and PCA-FRF show that the ANN trained with the proposed damage index of PCA-TSD in this research has more accurate results than the ANN trained with the common damage index of PCA-FRF.

-The results clearly indicate the efficiency of the network ensemble trained with the proposed combination of two compressed vibration data. The outcomes of the ensemble provide more accurate results than those of the two former individual neural networks.

-Although ANN ensemble is not a new technique for damage detection, the proposed ANN ensemble which is based on combination of two different damage indices is an innovative structural damage detection methodology with the high quality.

-The results of the ANN ensembles show that the proposed method is robust, reliable and precise in identifying defects of the braces of the CFS wall. The results also indicate the efficiency of the neural network ensemble approach, which gives outputs that are more accurate than any of the outputs of the individual neural networks 


\section{References}

Adams, R., P. Cawley, C. Pye and B. Stone. 1978. A vibration technique for non-destructively assessing the integrity of structures. Journal of Mechanical Engineering Science. 20(2): 93-100.

Bakhary, N., H. Hao and A. J. Deeks. 2007. Damage detection using artificial neural network with consideration of uncertainties. Engineering Structures. 29(11): 2806-2815.

Bakhary, N., H. Hao and A. J. Deeks. 2010. Structure damage detection using neural network with multi-stage substructuring. Advances in Structural Engineering. 13(1): 95-110.

Chen, H., C. Spyrakos and G. Venkatesh. 1995. Evaluating structural deterioration by dynamic response. Journal of Structural Engineering. 121(8): 1197-1204.

Dackermann, Ulrike, Jianchun Li, and Bijan Samali. 2010. Dynamic-based damage identification using neural network ensembles and damage index method. Advances in Structural Engineering. 13 (6): 1001-1016.

De Boe, P. and J.-C. Golinval. 2003. Principal component analysis of a piezosensor array for damage localization. Structural health monitoring. 2(2): 137-144.

Farrar, C. R., S. W. Doebling and D. A. Nix. 2001. Vibration-based structural damage identification. Philosophical Transactions of the Royal Society of London. Series A: Mathematical, Physical and Engineering Sciences. 359(1778): 131-149.

Ghaboussi, J. and X. Wu (1998). Soft computing with neural networks for engineering applications: Fundamental issues and adaptive approaches. Structural Engineering and Mechanics. 6(8): 955-969.

Hansen, L. K. and P. Salamon. 1990. Neural network ensembles. IEEE Transactions on Pattern Analysis \& Machine Intelligence. 12: 993-1001. 
Kim, J.-T., J.-H. Park and B.-J. Lee. 2007. Vibration-based damage monitoring in model plategirder bridges under uncertain temperature conditions. Engineering Structures. 29(7): 1354-1365. Koh, C., Y. Chen and C.-Y. Liaw. 2003. A hybrid computational strategy for identification of structural parameters. Computers \& Structures. 81(2): 107-117.

Kullaa, J. 2003. Damage detection of the Z24 bridge using control charts. Mechanical Systems and Signal Processing. 17(1): 163-170.

Lee, J. J., J. W. Lee, J. H. Yi, C. B. Yun and H. Y. Jung. 2005. Neural networks-based damage detection for bridges considering errors in baseline finite element models. Journal of Sound and Vibration. 280(3): 555-578.

Li, J., U. Dackermann, Y. L. Xu and B. Samali. 2011. Damage identification in civil engineering structures utilizing PCA-compressed residual frequency response functions and neural network ensembles. Structural Control and Health Monitoring. 18(2): 207-226.

Li, Z.-X. and X.-M. Yang. 2008. Damage identification for beams using ANN based on statistical property of structural responses. Computers \& structures. 86(1): 64-71.

Mazzoni, S., F. McKenna, M. H. Scott and G. L. Fenves. 2006. OpenSees command language manual. Pacific Earthquake Engineering Research (PEER) Center.

Ni, Y., X. Zhou and J. Ko. 2006. Experimental investigation of seismic damage identification using PCA-compressed frequency response functions and neural networks. Journal of Sound and Vibration. 290(1): 242-263.

Rytter, A. 1993. Vibrational based inspection of civil engineering structures.

Shu, J., Z. Zhang, I. Gonzalez and R. Karoumi. 2013. The application of a damage detection method using Artificial Neural Network and train-induced vibrations on a simplified railway bridge model. Engineering structures. 52: 408-421. 
Tang, J. 2005. Frequency response based damage detection using principal component analysis. Information Acquisition. 2005 IEEE International Conference on, IEEE.

Trendafilova, I. 2005. A study on vibration-based damage detection and location in an aircraft wing scaled model. Applied Mechanics and Materials, Trans Tech Publ. 3: 309-314.

Worden, K., G. Manson and N. Fieller. 2000. Damage detection using outlier analysis. Journal of Sound and Vibration. 229(3): 647-667.

Zang, C. and M. Imregun. 2001. Structural damage detection using artificial neural networks and measured FRF data reduced via principal component projection. Journal of Sound and Vibration. 242(5): 813-827.

Zeynalian, M. and H. Ronagh. 2012. A numerical study on seismic performance of strap-braced cold-formed steel shear walls. Thin-walled structures. 60: 229-238.

Zhou, Z.-H., J. Wu and W. Tang. 2002. Ensembling neural networks: many could be better than all. Artificial intelligence. 137(1): 239-263. 
Table 1: Mechanical properties of the C section (Zeynalian and Ronagh 2012)

\begin{tabular}{cccccc}
\hline No. & Property & Value & No. & Property & Value \\
\hline \hline 1 & Nominal grade & $550 \mathrm{MPa}$ & 5 & Yield strain & $0.45 \%$ \\
\hline 2 & Nominal thickness & $0.55 \mathrm{~mm}$ & 6 & Ultimate stress $F_{u}$ & $617 \mathrm{MPa}$ \\
\hline 3 & Elastic modulus & $169 \mathrm{GPa}$ & 7 & Ultimate strain & $2.86 \%$ \\
\hline 4 & Yield stress $F_{y}$ & $592 \mathrm{MPa}$ & 8 & $F_{u} / F_{y}$ & 1.04 \\
\hline
\end{tabular}


Table 2: Mechanical properties of the straps (Zeynalian and Ronagh 2012)

\begin{tabular}{cccccc}
\hline No. & Property & Value & No. & Property & Value \\
\hline \hline 1 & Nominal grade & $300 \mathrm{MPa}$ & 4 & Yield stress $F_{y}$ & $310 \mathrm{MPa}$ \\
\hline 2 & Nominal thickness & $0.8 \mathrm{~mm}$ & 5 & Yield strain & $0.18 \%$ \\
\hline 3 & Elastic modulus & $163 \mathrm{GPa}$ & 6 & Ultimate stress $F_{u}$ & $370 \mathrm{MPa}$ \\
\hline
\end{tabular}


Table 3. Damage scenarios of the CFS shear wall

\begin{tabular}{|c|c|c|c|}
\hline Damage scenario & $\begin{array}{c}\text { Stiffness Reduction } \\
\text { of brace } 1\end{array}$ & $\begin{array}{c}\text { Stiffness Reduction } \\
\text { of brace } 2\end{array}$ & SSR \\
\hline 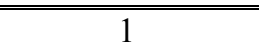 & 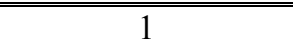 & $\bar{~} 1$ & 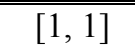 \\
\hline 2 & 1 & 0.9 & {$[1,0.9]$} \\
\hline 3 & 1 & 0.8 & {$[1,0.8]$} \\
\hline$\ldots$ & $\ldots$ & $\ldots$ & $\ldots$ \\
\hline 9 & 1 & 0.2 & {$[1,0.2]$} \\
\hline 10 & 0.9 & 1 & {$[0.9,1]$} \\
\hline 11 & 0.9 & 0.9 & {$[0.9,0.9]$} \\
\hline 12 & 0.9 & 0.8 & {$[0.9,0.8]$} \\
\hline$\cdots$ & $\cdots$ & $\ldots$ & $\cdots$ \\
\hline 80 & 0.2 & 0.3 & {$[0.2,0.3]$} \\
\hline 81 & 0.2 & 0.2 & {$[0.2,0.2]$} \\
\hline
\end{tabular}


Table 4: Modal parameter changes for different damage severities

\begin{tabular}{|c|c|c|c|c|c|c|}
\hline \multirow{2}{*}{$\begin{array}{l}\text { Mode } \\
\text { shape }\end{array}$} & \multirow{2}{*}{$\begin{array}{l}\text { Damage } \\
\text { feature }\end{array}$} & \multicolumn{5}{|c|}{ SSR } \\
\hline & & {$[1,1]$} & {$[0.85,0.85]$} & {$[0.45,0.45]$} & {$[0.25,0.25]$} & {$[0.35,0.75]$} \\
\hline \multirow{3}{*}{1} & $\overline{\mathrm{NF}}$ & 81.4 & 79.2 & 60.8 & "45.7 & 66.6 \\
\hline & $\begin{array}{l}\text { MSR in } \\
\text { place } 1\end{array}$ & 1 & 0.80 & 0.25 & 0.17 & 0.32 \\
\hline & $\begin{array}{l}\text { MSR in } \\
\text { place } 2\end{array}$ & 1 & 1.60 & 2.30 & 2.33 & 2.27 \\
\hline \multirow{3}{*}{2} & $\mathrm{NF}$ & 93.0 & 88.5 & 84.7 & 84.3 & 85.0 \\
\hline & $\begin{array}{l}\text { MSR in } \\
\text { place } 1\end{array}$ & 1 & 1.65 & 2.31 & 2.40 & 2.31 \\
\hline & $\begin{array}{l}\text { MSR in } \\
\text { place } 2\end{array}$ & 1 & 0.87 & 0.27 & 0.18 & 0.35 \\
\hline
\end{tabular}


Table 5: Information of individual neural networks

\begin{tabular}{cc}
\hline Neural network & $\begin{array}{c}\text { Numbers of neurons } \\
\text { (input layer, hidden layer, output layer) }\end{array}$ \\
\hline \hline NF & $2 ; 5 ; 1$ \\
\hline MF+MSR & $4 ; 10 ; 1$ \\
\hline CNR-FRF & $6 ; 15 ; 1$ \\
\hline COV-TSD & $3 ; 7 ; 1$ \\
\hline PCA-compressed FRF & $4 ; 10 ; 1$ \\
\hline
\end{tabular}


Table 6: Percentage of false outputs for individual networks-based damage detection of brace 1 .

\begin{tabular}{ccccccc}
\hline & \multicolumn{7}{c}{ Damage index } \\
\cline { 2 - 7 } Noise intensity & NF & MF+MSR & CNR-FRF & COV-TSD & PCA-FRF & PCA-TSD \\
\hline \hline $1 \%$ & 86.4 & 80.2 & 57.2 & 72.8 & 7.9 & 3.0 \\
\hline $3 \%$ & 86.8 & 78.6 & 63.8 & 73.3 & 30.2 & 15.8 \\
\hline $5 \%$ & 86.4 & 81.9 & 58.8 & 77.0 & 45.4 & 25.9 \\
\hline
\end{tabular}


Table 7: Average value of absolute normalized error for individual networks-based damage detection of brace1.

\begin{tabular}{|c|c|c|c|c|c|c|}
\hline \multirow[b]{2}{*}{ Noise intensity } & \multicolumn{6}{|c|}{ Damage index } \\
\hline & NF & $\mathrm{MF}+\mathrm{MSR}$ & CNR-FRF & COV-TSD & PCA- FRF & PCA- TSD \\
\hline $1 \%$ & 22.5 & 43.9 & 10.5 & 15.3 & 3.6 & 1.5 \\
\hline $3 \%$ & 23.1 & 48.9 & 10.7 & 15.3 & 7.2 & 4.9 \\
\hline $5 \%$ & 23.5 & 53.6 & 10.6 & 16.1 & 10.9 & 7.0 \\
\hline
\end{tabular}


Table 8: Maximum value of absolute normalized error for individual networks-based damage detection of brace 1 .

\begin{tabular}{|c|c|c|c|c|c|c|}
\hline \multirow[b]{2}{*}{ Noise intensity } & \multicolumn{6}{|c|}{ Damage index } \\
\hline & NF & $\mathrm{MF}+\mathrm{MSR}$ & CNR-FRF & COV-TSD & PCA- FRF & PCA- TSD \\
\hline $1 \%$ & 48.2 & 19.0 & 31.5 & 46.5 & 7.1 & 5.2 \\
\hline $3 \%$ & 52.5 & 18.9 & 25.7 & 48.0 & 16.9 & 14.7 \\
\hline $5 \%$ & 46.7 & 19.2 & 24.4 & 51.2 & 30.0 & 28.3 \\
\hline
\end{tabular}


Table 9: Percentage of false outputs for individual networks-based damage detection of brace 2.

\begin{tabular}{ccccccc}
\hline & \multicolumn{7}{c}{ Damage index } \\
\cline { 2 - 7 } Noise intensity & NF & MF+MSR & CNR-FRF & COV-TSD & PCA- FRF & PCA- TSD \\
\hline \hline $1 \%$ & 85.2 & 80.2 & 56.0 & 75.7 & 8.4 & 5.1 \\
\hline $3 \%$ & 85.6 & 75.7 & 67.5 & 77.8 & 29.2 & 14.2 \\
\hline $5 \%$ & 86.4 & 77.4 & 70.0 & 79.4 & 43.8 & 28.1 \\
\hline
\end{tabular}


Table 10: Average value of absolute normalized error for individual networks-based damage detection of brace 2 .

\begin{tabular}{ccccccc}
\hline & \multicolumn{7}{c}{ Damage index } \\
\cline { 2 - 7 } Noise intensity & NF & MF+MSR & CNR-FRF & COV-TSD & PCA- FRF & PCA- TSD \\
\cline { 2 - 7 } & & & & & & \\
\hline & 22.9 & 18.9 & 9.7 & 16.4 & 2.9 & 2.5 \\
\hline $3 \%$ & 22.4 & 18.5 & 11.5 & 16.9 & 6.1 & 5.0 \\
\hline $5 \%$ & 22.2 & 18.7 & 12.1 & 17.3 & 11.3 & 8.4 \\
\hline
\end{tabular}


Table 11: Maximum value of absolute normalized error for individual networks-based damage detection of brace 2 .

\begin{tabular}{|c|c|c|c|c|c|c|}
\hline \multirow[b]{2}{*}{ Noise intensity } & \multicolumn{6}{|c|}{ Damage index } \\
\hline & NF & $\mathrm{MF}+\mathrm{MSR}$ & CNR-FRF & COV-TSD & PCA- FRF & PCA- TSD \\
\hline $1 \%$ & 43.9 & 245.8 & 32.2 & 244.7 & 9.1 & 4.4 \\
\hline $3 \%$ & 53.9 & 43.3 & 36.9 & 51.1 & 15.5 & 20.1 \\
\hline $5 \%$ & 48.5 & 52.6 & 44.2 & 54.5 & 32.8 & 29.3 \\
\hline
\end{tabular}


Table 12: Results of network ensemble trained with the collection of two PCA- compressed vibration data.

\begin{tabular}{lcccccc}
\hline & \multicolumn{3}{c}{ Strap brace 1 } & \multicolumn{2}{c}{ Strap brace 2 } \\
Noise intensity & $1 \%$ & $3 \%$ & $5 \%$ & $1 \%$ & $3 \%$ & $5 \%$ \\
\hline \hline Percentage of false detection & 0 & 0 & 6 & 0 & 3 & 7.8 \\
\hline $\begin{array}{l}\text { Maximum value of absolute } \\
\text { normalized error }\end{array}$ & 2.08 & 4.94 & 9.76 & 4.67 & 12.97 & 14.92 \\
\hline $\begin{array}{l}\text { Average value of absolute } \\
\text { normalized error }\end{array}$ & 0.78 & 1.51 & 2.39 & 1.14 & 2.09 & 2.81 \\
\hline
\end{tabular}




\section{List of Figure Captions}

Figure 1: The strap-braced CFS shear wall (Zeynalian and Ronagh 2012).

Figure 2: FE model of the CFS shear wall.

Figure 3: Elastic pushover curves of the CFS shear wall.

Figure 4: FRF summation graphs for the healthy and damage states of the CFS shear wall.

Figure 5: The relationship between change of the variance (measuring point 2) and the damage scenarios.

Figure 6: Individual and cumulative contribution of the first 50 PCs of the summation of residual FRFs of the shear wall.

Figure 7: Schematic model of implemented neural network ensemble trained with a collection of PCA-compressed FRFs and acceleration responses.

Figure 8: Flow chart of the implemented neural network ensemble for multi-damage detection of strap braces of CFS shear wall.

Figure 9: Damage severity outcomes of neural network ensemble trained with a collection of PCA-compressed FRFs and acceleration responses. 


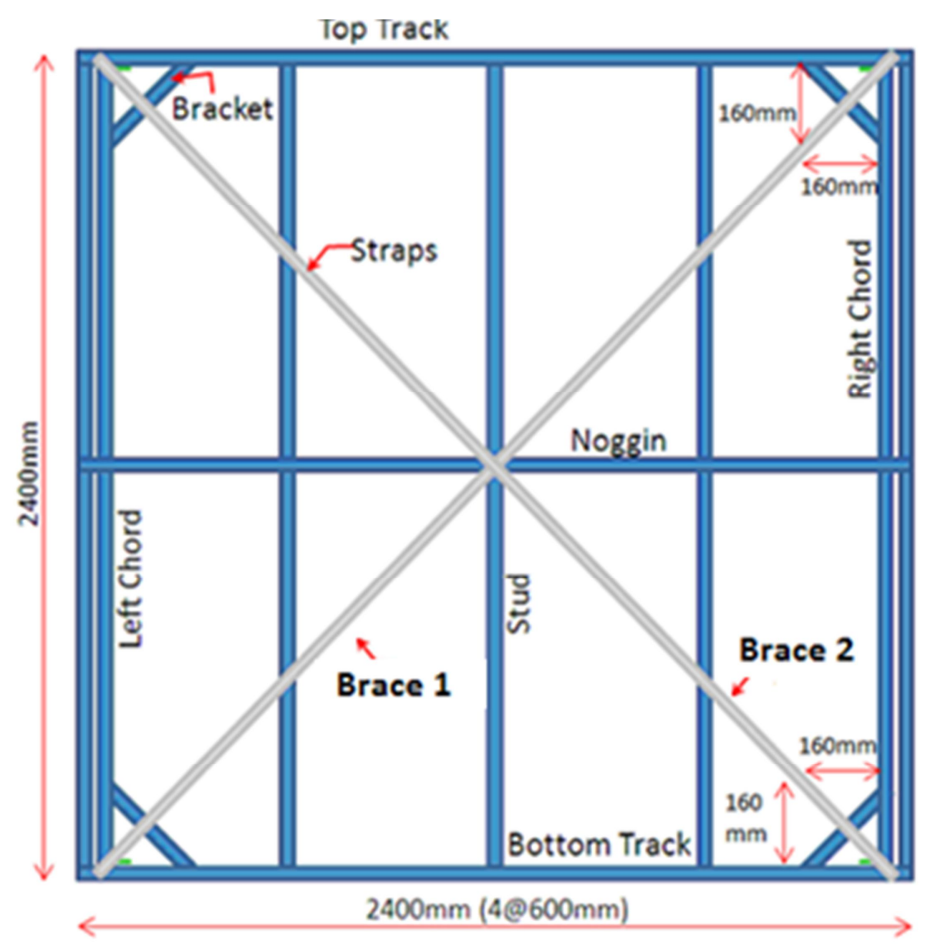

https://mc06.manuscriptcentral.com/cjce-pubs 


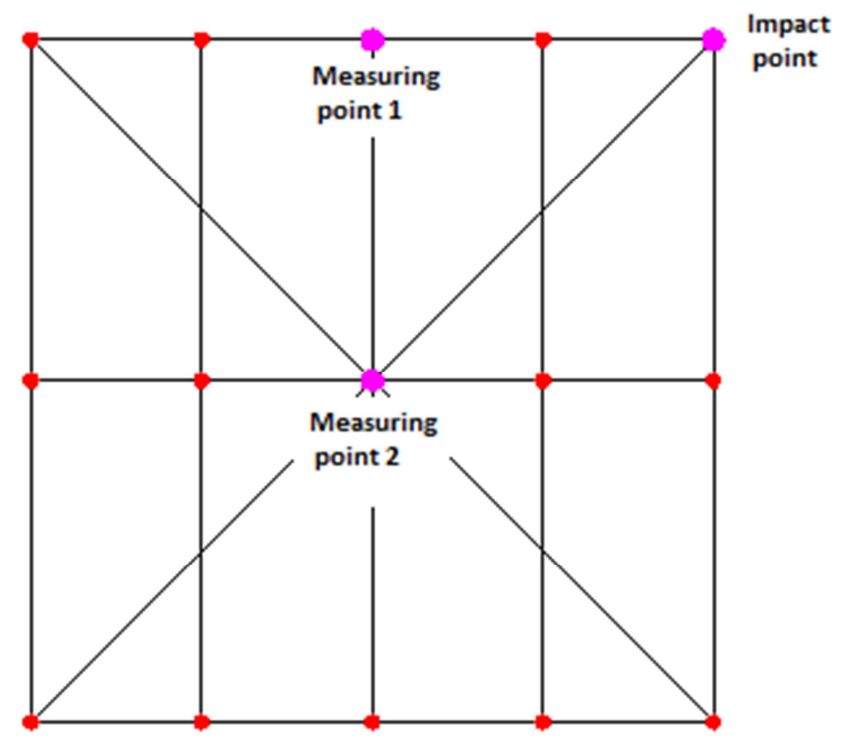




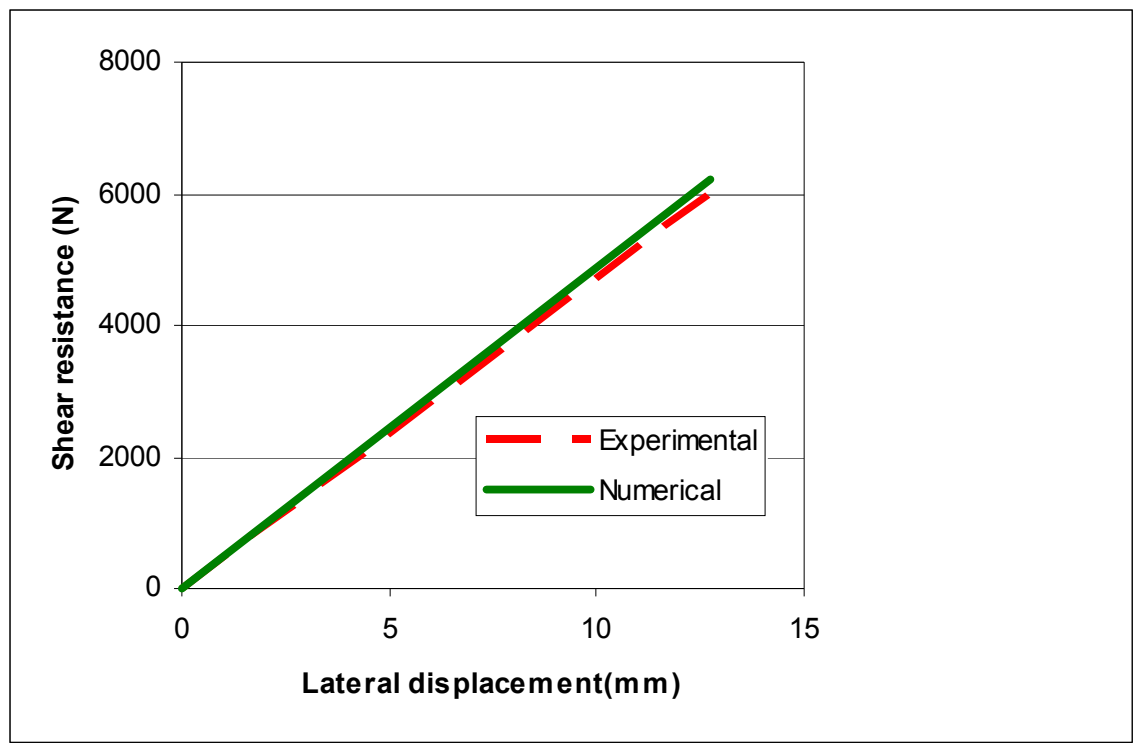




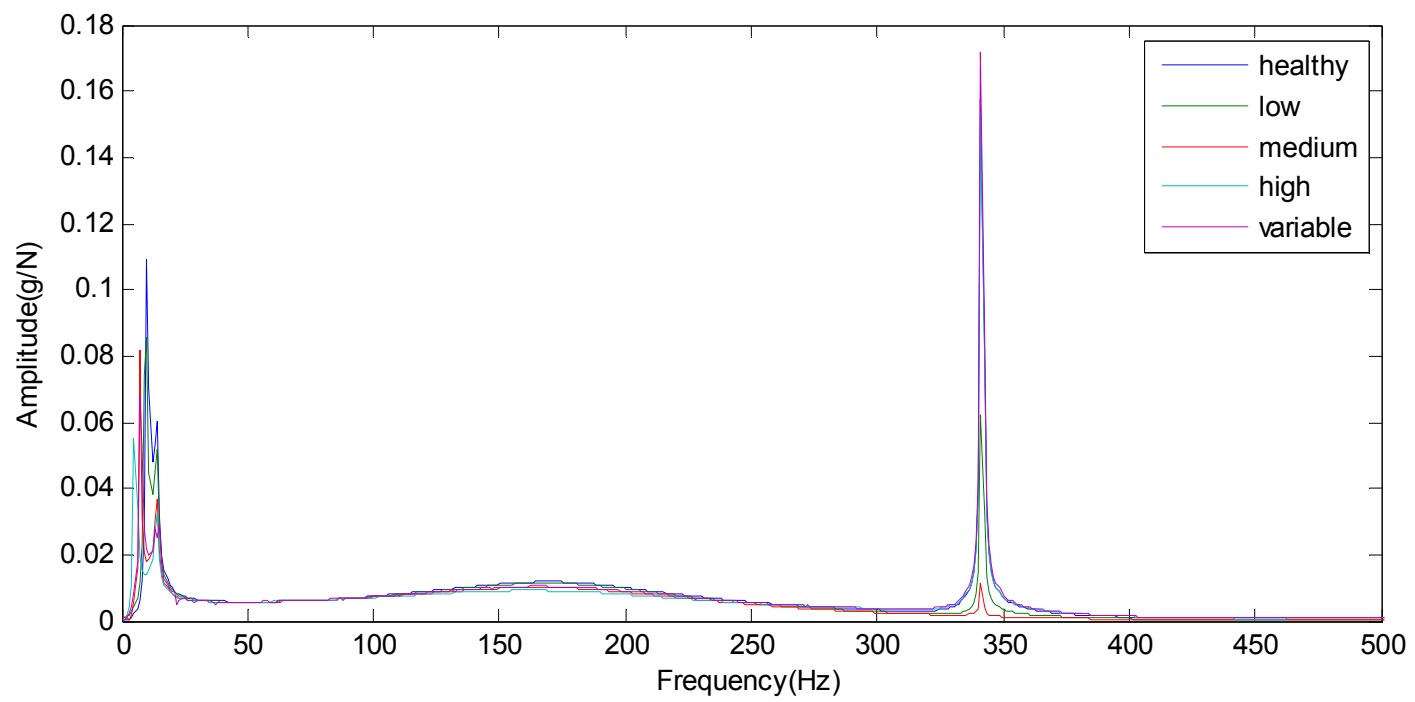




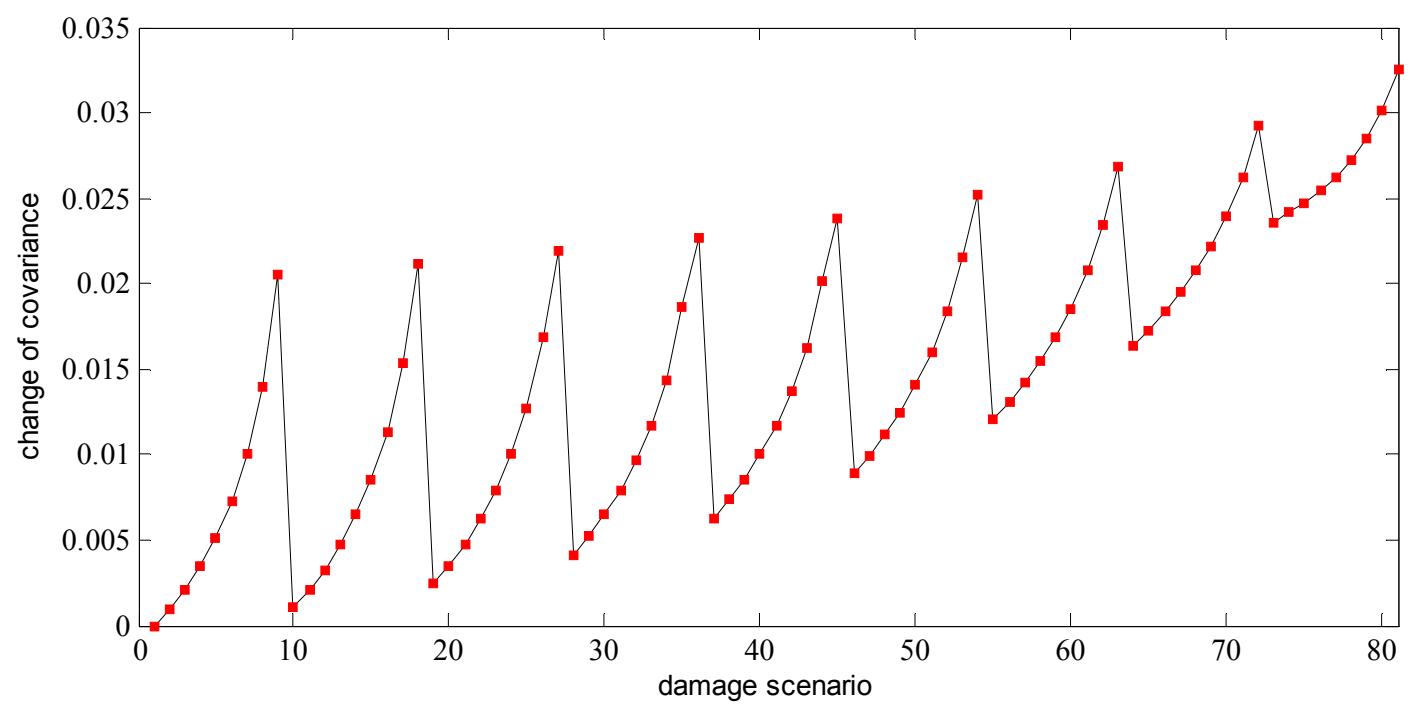




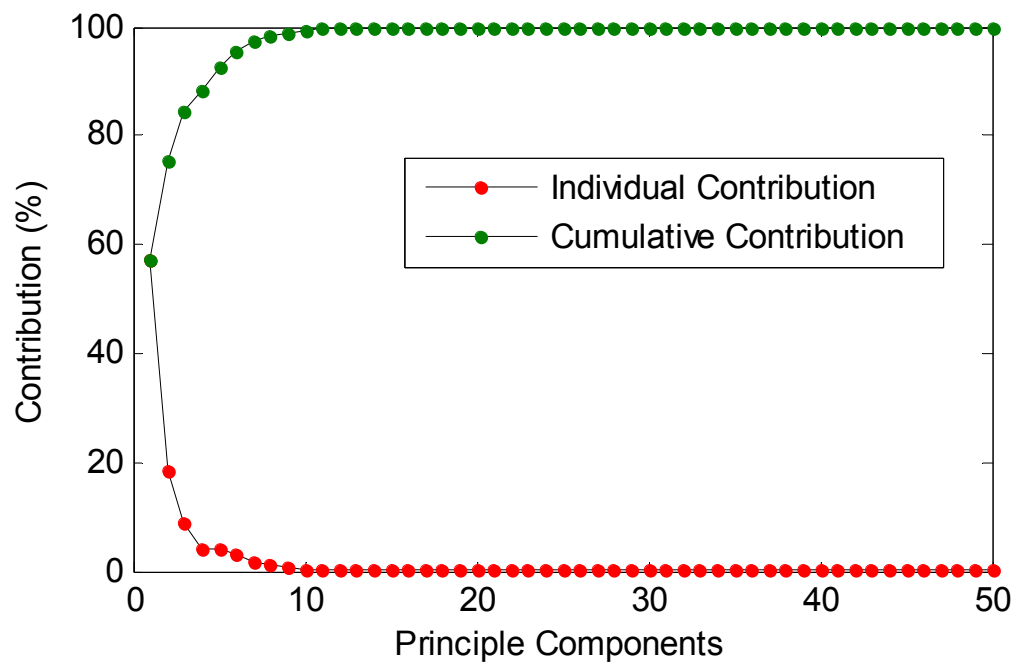

https://mc06.manuscriptcentral.com/cjce-pubs 


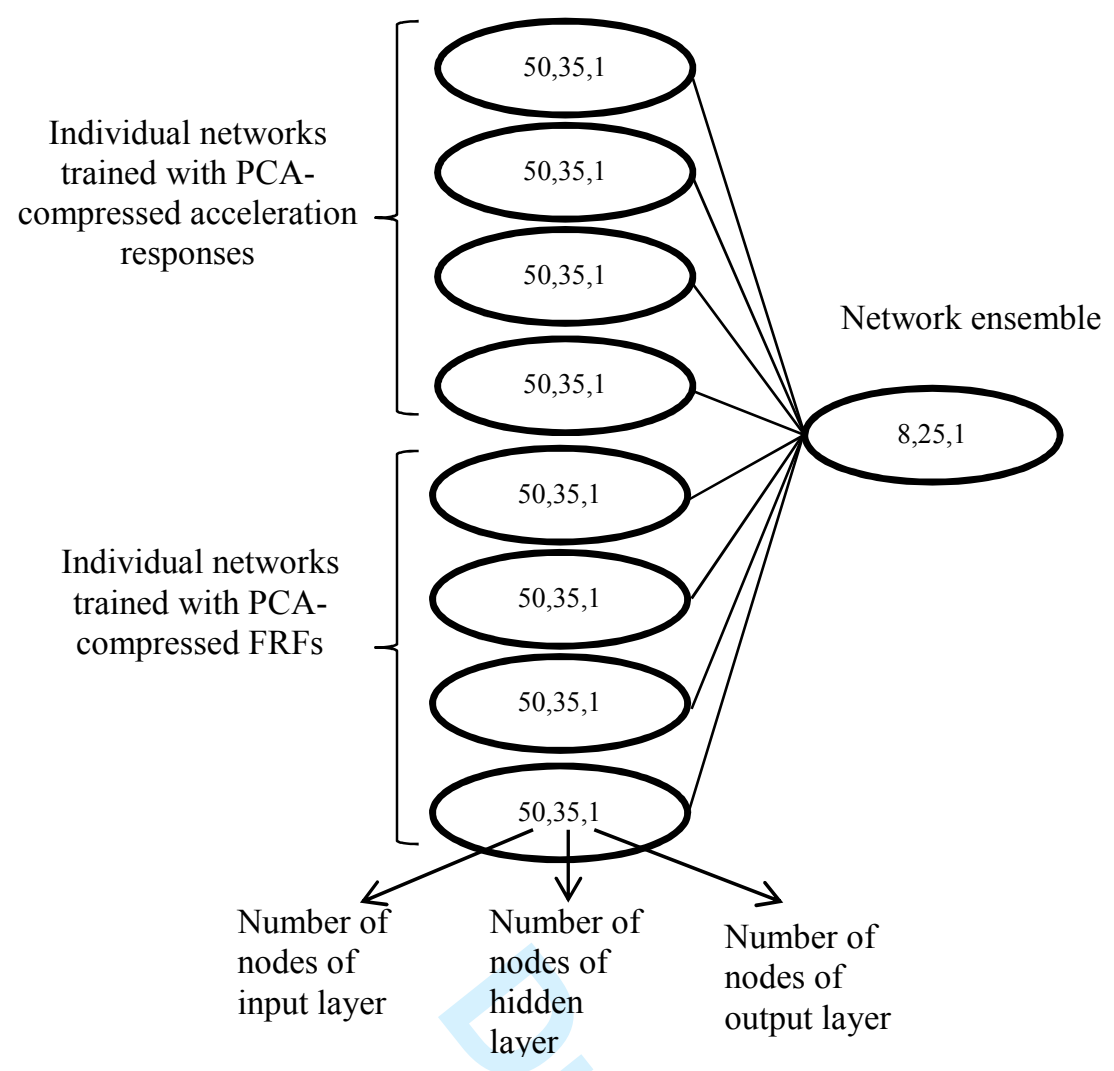




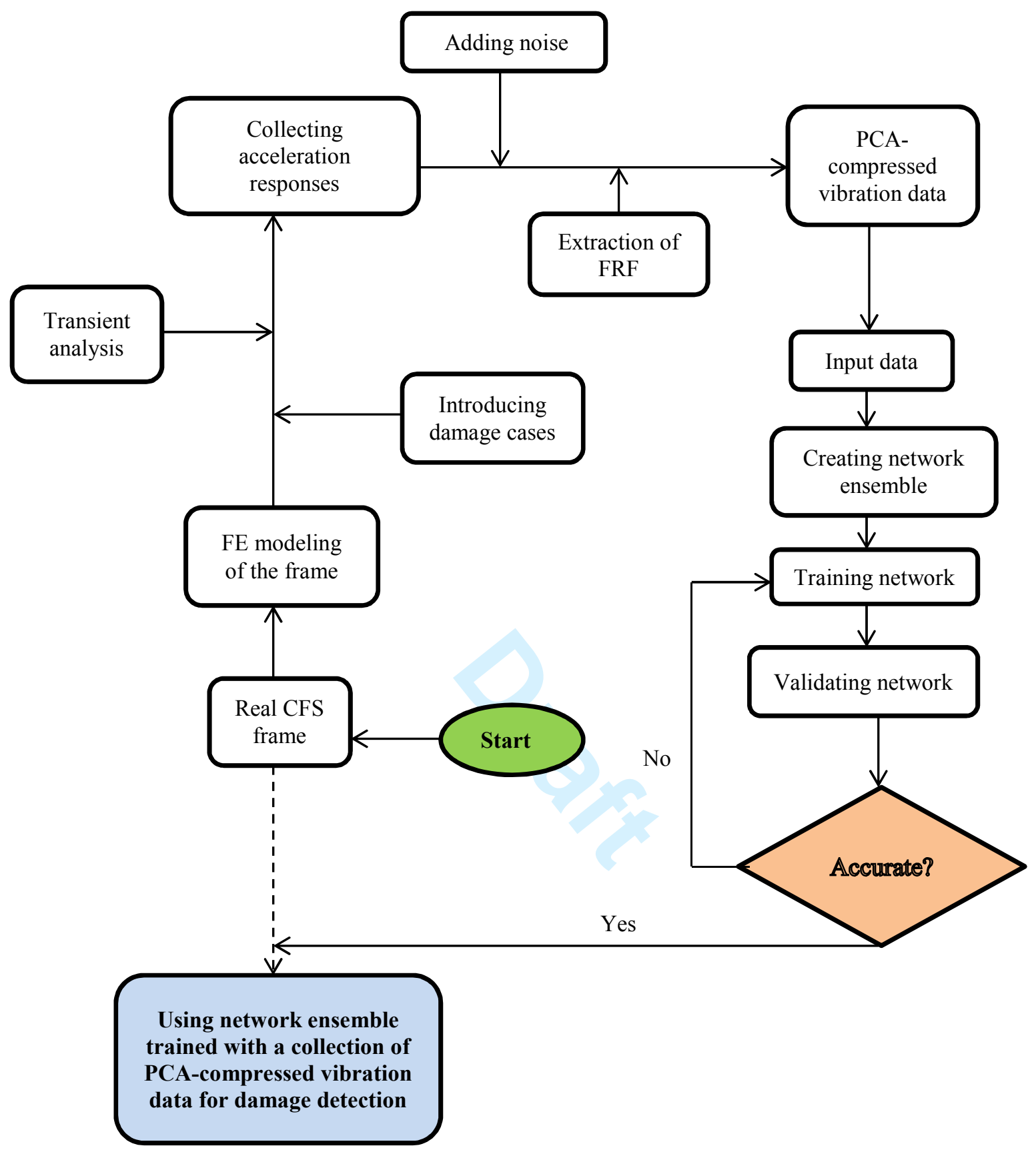




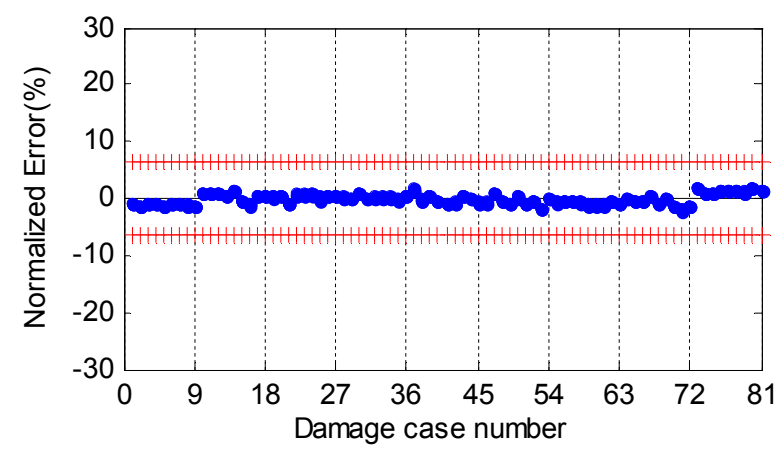

(a)For strap brace 1 with $1 \%$ noise pollution

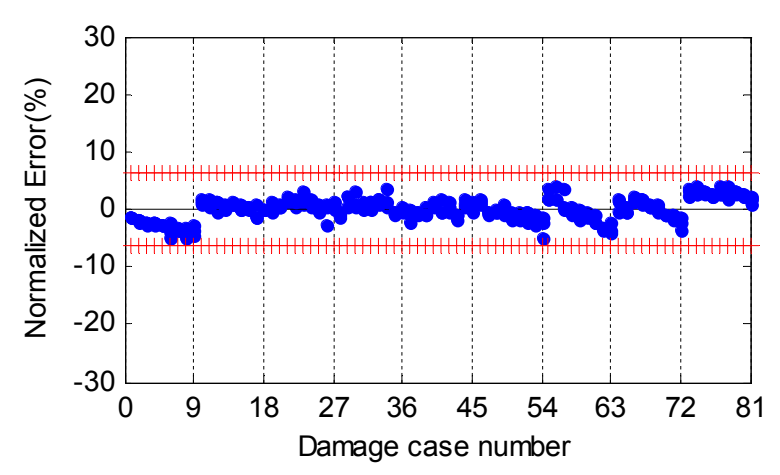

(c)For strap brace 1 with 3\% noise pollution

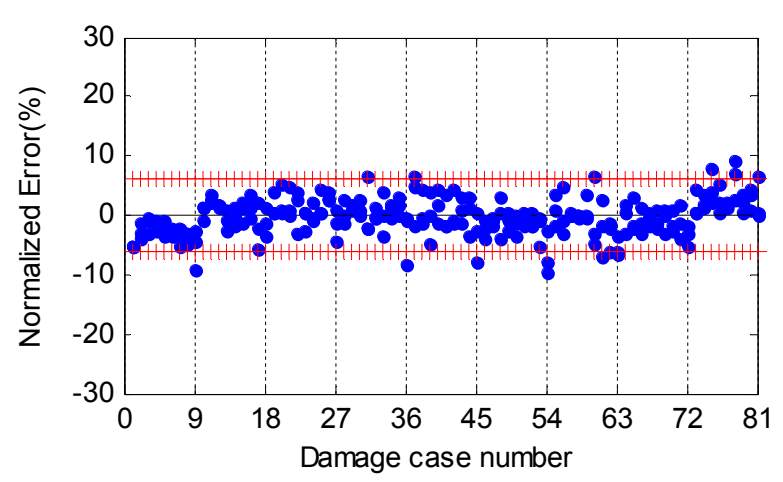

(e)For strap brace 1 with 5\% noise pollution

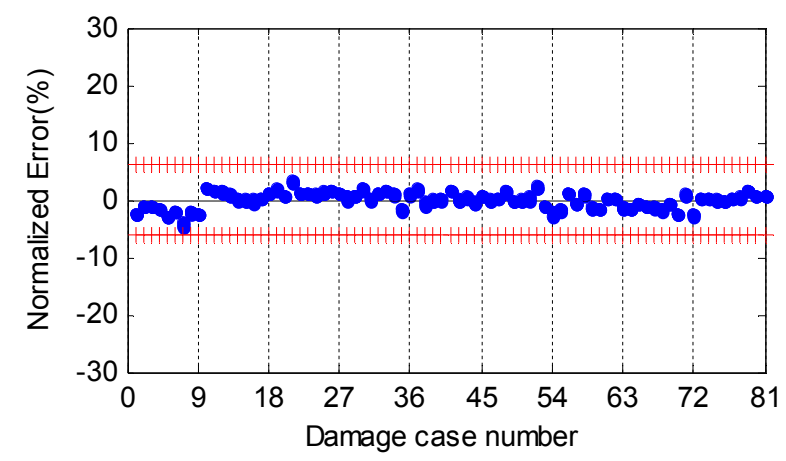

(b)For strap brace 2 with $1 \%$ noise pollution

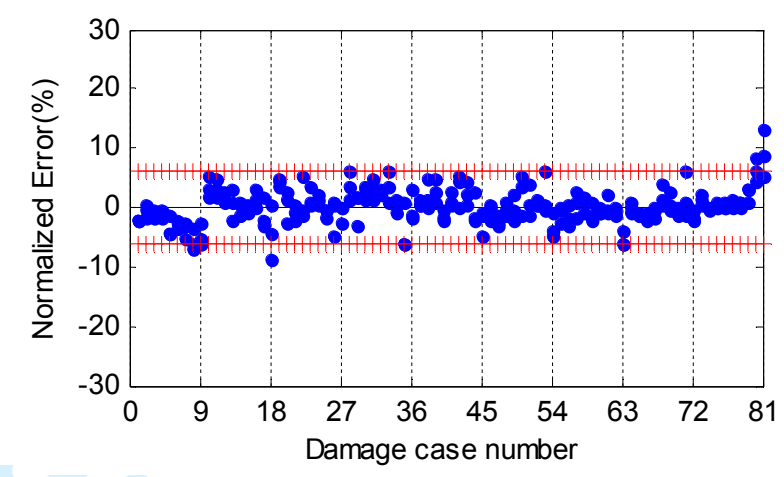

(d)For strap brace 2 with 3\% noise pollution

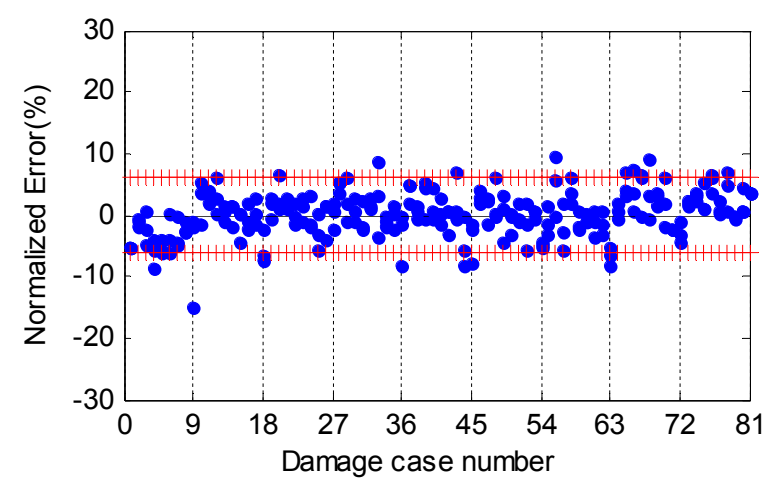

(f) For strap brace 2 with 5\% noise pollution 